\title{
Hereditary spastic paraplegia associated with epilepsy, mental retardation and hearing impairment
}

\author{
J S Yih MD, Shuu-Jiun Wang MD, Ming-Shung Su MD, Shi-Ching Tsai MD, \\ Ryh-Huei Lin MD, Ker-Neng Lin MS, Hsiu-Chih Liu MD \\ The Neurological Institute, Veterans General Hospital-Taipei, and National Yang-Ming \\ Medical College, Taipei, Taiwan, Republic of China.
}

Epilepsy rarely occurs in patients with hereditary spastic paraplegia (HSP), and is not included in the description of the 'complicated' form of HSP by Harding. ${ }^{2}$ We report 3 patients with HSP in a family of two generations. Two of them also had epilepsy, mental retardation and hearing impairment. The disorder was inherited as an autosomal dominant trait.

Keywords: hereditary diseases; spastic paraplegia; epilepsy.

\section{Introduction}

Hereditary spastic paraplegia (HSP) is a rare inherited neurodegenerative disorder characterised primarily by progressive spasticity and weakness of legs. ${ }^{1}$ Harding $^{2}$ divided HSP into 'pure' and 'complicated' forms. The complicated form included other neurological abnormalities, but epilepsy was not one of them. There have been few reports of the association of epilepsy and HSP. ${ }^{3-5}$ We report HSP in three members of a family in two generations. Two of them also had epilepsy, mental retardation and hearing impairment.

\section{Case reports}

\section{Family history}

This family included three children and their parents. The marriage was not consanguineous. The father is healthy and his parents and siblings do not have neurological disorders. The mother was a 37 year old woman who suffered from spastic paraplegia since age 5 . Her mother also had mild gait disturbance, and died in an accident. The eldest daughter, 19 years old, was adopted and is healthy. The other two children suffered from spastic paraplegia and epilepsy, as is shown in the family pedigree (Fig 1).

\section{Case 1}

This 17 year old boy was the product of a full term pregnancy and normal delivery. At age 6, he began to have weakness of the legs which

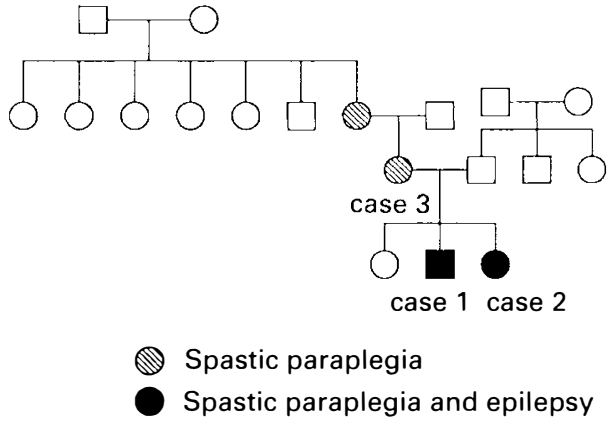

Figure 1 Pedigree of the family.

gradually progressed. At age 8 , he began to have epileptic seizures characterised by loss of consciousness and clonic convulsion of both upper limbs lasting 2-3 minutes. Initially, the epileptic seizures occurred once or twice per year but at age 15 they became more frequent, up to twice a week. At age 17, he developed another pattern of seizures. He showed lapse of consciousness with atonic head nodding for 2-3 seconds with quick recovery. Myoclonic jerks of both upper limbs also occurred independently.

On examination at age 15 , strength was intact in his upper limbs, but there was grade 4 weakness of lower limbs. Deep tendon reflexes were normal in the upper limbs, but the knee and ankle jerks were $4+$ bilaterally. There was severe spasticity of both legs and he walked with a scissors gait and required assistance for walking. Babinski's sign was present bilaterally. Finger-to-nose test was normal bilaterally. Rapid alternating movements of both hands were normal. Heel-to-shin test was clumsy on 
both sides because of spasticity. All modalities of sensation were normal. Fundoscopic examination showed no pigmentary retinopathy. His gait and the strength of his legs have been progressively deteriorating and he has been wheelchair bound since age 16 .

At age 8, the electroencephalogram (EEG) revealed multifocal spikes with a posterior background of $8 \mathrm{~Hz}$ alpha rhythm. At age 15 , the EEG showed $7-8 \mathrm{~Hz}$ rhythmic waves over the posterior scalp regions. Small amount of $2-4 \mathrm{~Hz}$ slow waves were widely distributed and frontal intermittent rhythmic delta activity (FIRDA) of $2 \mathrm{~Hz}$ occurred rarely. There were paroxysmal bursts of generalised polyspikes or polyspike-and-wave complexes repeating at $3 \mathrm{~Hz}$ and lasting for 1-2 seconds. At age 17, the posterior background of the EEG deteriorated to $6-7 \mathrm{~Hz}$. Diffuse $2-4 \mathrm{~Hz}$ slow waves and $2.5 \mathrm{~Hz}$ FIRDA were much increased. Active generalised polyspikes and polyspike-and-wave complexes were also recorded (Fig 2).

Nerve conduction studies (NCS) were normal. Pure tone audiogram disclosed moderate sensorineural hearing loss bilaterally. IQ testing showed moderate mental retardation, with verbal IQ 52, performance IQ 57 and full scale IQ 50. A CT scan of the brain was normal. Antibodies to HTLV-I in serum were negative.

\section{Case 2}

A 14 year old girl, the younger sister of case 1, suffered from progressive spastic weakness since age 5. She had no seizures until age 14 when she began to have several attacks of head turning toward the right and clonic convulsion of both upper limbs lasting 1-2 minutes, followed by drowsiness for 10 minutes. She had no recollection of the attacks.

On examination, her strength was normal in both upper limbs, but there was mild weakness of both legs with severe spasticity. Ankle jerks and knee jerks were hyperreflexic. Deep tendon reflexes in the upper limbs were normal. Babinski's sign was present bilaterally. She had a scissors gait and needed assistance for walking. Finger-to-nose tests and rapid alternating movements were normal. Heel-to-shin test was clumsy bilaterally due to spasticity of the legs. All sensory modalities were normal. There was no pigmentary retinopathy on fundoscopic examination.

EEG revealed multifocal spikes especially over the centroparietal areas bilaterally. The posterior background consisted of $8-9 \mathrm{~Hz}$ rhythmic waves with poor regularity, and a small amount of medium amplitude $4-7 \mathrm{~Hz}$ theta waves were widely distributed.

NCS was normal. Pure tone audiogram revealed mild to moderate sensorineural hearing

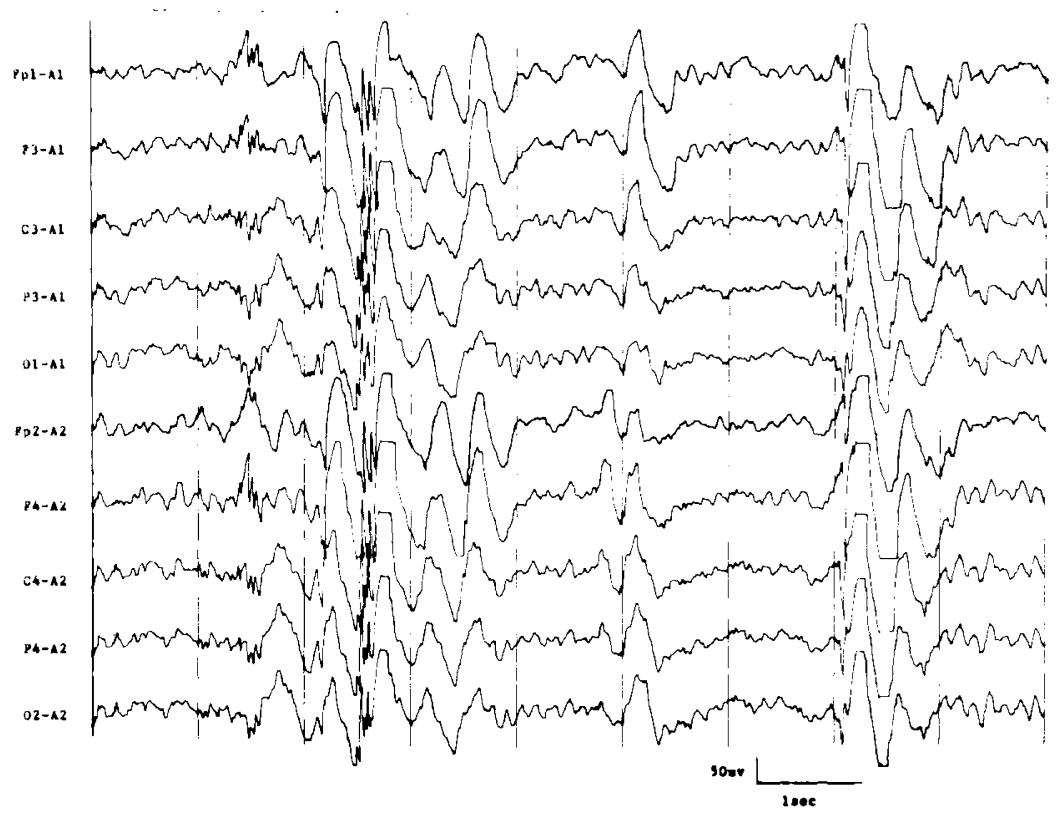

Figure 2 Electroencephalogram of case 1 shows generalised polyspike and polyspike-and-wave complexes and frontal intermittent rhythmic delta activity. 
loss bilaterally. IQ test disclosed moderate mental retardation, with verbal IQ 55, performance IQ 68 and full scale IQ 57. CT scan of brain was normal. Serum antibodies to HTLV-I were negative.

\section{Case 3}

This 37 year old woman, the mother of cases 1 and 2 , has had progressive spastic weakness of both legs since age 2 and had to hold on to objects to walk since age 21 . Her intelligence was subnormal. There was marked spasticity and mild weakness of the legs. She had a scissors gait, and required assistance. Ankle jerks were $3+$, but the knee jerks could not be elicited. Sensation was intact. Fundoscopic examination did not reveal pigmentary retinopathy. EEG showed poorly sustained $9 \mathrm{~Hz}$ alpha rhythm in the posterior background and much accentuated beta activity.

\section{Discussion}

This family has a disease characterised by spastic paraplegia, generalised seizure disorder, mental retardation and hearing impairment. Although the spastic paraplegia was progressive, the mental retardation of the brother and sister and the subnormal intelligence of the mother have been a persistent abnormality without progression. Mental retardation and hearing impairment are known to be associated with HSP. ${ }^{2,6,7}$ Epilepsy is rarely associated with HSP and is not included in the 'complicated' form of HSP by Harding's classification. ${ }^{2}$ In our family, both siblings have generalised seizures and EEG changes. The brother developed generalised clonic seizures 2 years after the onset of spastic paraplegia. His seizures gradually became more frequent, atonic seizures and myoclonic jerks developed later, and the EEG findings also deteriorated. The sister developed seizures 9 years after the onset of spastic paraplegia. Her seizures are less frequent than are those of her brother. Long term follow up is necessary to determine whether her seizure attacks and EEG findings will deteriorate as did her brother's.

Since epilepsy is a common disorder and genetic factors play an important role in its occurrence, ${ }^{8}$ one could raise the objection that epilepsy and HSP are coincidental findings in this family. We believe the possibility of two separate genetic conditions (HSP and epilepsy) occurring in the same family is unlikely, and that it is more reasonable to regard the HSP and epilepsy as the features of one genetic disease. There have been a few previous reports of the association of epilepsy and HSP. ${ }^{3-5}$ Bruyn and Mechelse ${ }^{3}$ reported that one of their 3 cases of HSP had petit mal and characteristic EEG findings. Four other members of the pedigree also had EEG changes consistent with epilepsy. Kuroda et al $^{4}$ described a man and his sister who developed features of HSP, 5 and 16 years respectively after the onset of primary generalised epilepsy with tonic-clonic seizures. One son of the sister had epilepsy. He suggested a close relationship between HSP and epilepsy. Sommerfelt et al ${ }^{5}$ reported 4 affected siblings in a family with HSP, epileptic myoclonus, mental retardation, hearing loss, ataxia and distal muscle atrophy. EEG in 3 cases showed epileptogenic activity, and polyspikes were seen in one of them.

Recently, Tedeschi et $a l^{9}$ have performed neurophysiological and neuropsychological tests in 11 patients with HSP ( 7 with the pure form and 4 with the complicated form). There was a high incidence of multisystem subclinical involvement of the central nervous system. He then proposed that HSP is a multisystem degenerative disease of the CNS. None of these patients had epilepsy, and EEG was not performed on any of them. EEG is not done routinely in patients with HSP, especially the pure form, because HSP is regarded as a spinal disease. We also believe that in HSP there is multisystem involvement of the CNS. EEG abnormalities may be found more often if EEG is done routinely on patients with HSP.

\section{Acknowledgements}

The study was supported in part by grants from the National Science Council (NSC 80-0412BO75-72) and Chin-Lin Medical Foundation (CI-80-25) 


\section{References}

1 Strumpell A (1880) Beitrage zur Pathologie des Ruckenmarks.; Arch Psychiat Nervenkr 10: 676-717.

2 Harding AE (1983) Classification of the hereditary ataxias and paraplegias. Lancet i: 8334: 1151-1155.

3 Bruyn GW, Mechelse K (1962) The association of familial spastic paraplegia and epilepsy in one family. Psychiatry Neurol Neurochir 65: 280-292.

4 Kuroda S (1985) Familial spastic paraplegia with epilepsy. Acta Med Okayama 39(2): 113-117.

5 Sommerfelt K, Lyllerman M, Sanner G (1991) Hereditary spastic paraplegia with epileptic myoclonus. Acta Neurol Scand 84: 157-160.

6 Fitzsimmons JS, Watson AR, Mellor D, Guilbert PR (1988) Familial spastic paraplegia, bilateral sensorineural deafness, and intellectural retardation associated with a progressive nephropathy. J Med Genet 25: 168-172.

7 Gustavson KH, Modrzewska K, Erikson A (1989) Hereditary spastic diplegia with mental retardation in two young siblings. Clin Genet 36: 439-441.

8 Niedermeyer E (1990) Epileptic seizure etiologies. In: Niedermeyer E, editor. The Epilepsies: Diagnosis and Management. Urban \& Schwarzenberg, Munich and Baltimore: 85-108.

9 Tedeschi G, Allocca S, Di Costanzo A, Carlomagno S, Merla F, Petretta V, et al (1991) Multisystem involvement of the central nervous system in Strumpell's disease. A neurophysiological and neuropsychological study. J Neurol Sci 103: 55-60. 